\title{
Nanotoxicology: characterizing the scientific literature, 2000-2007
}

\author{
Alexis D. Ostrowski - Tyronne Martin • \\ Joseph Conti · Indy Hurt • \\ Barbara Herr Harthorn
}

Received: 30 November 2008/ Accepted: 4 December 2008/Published online: 28 December 2008

(C) Springer Science+Business Media B.V. 2008

\begin{abstract}
Understanding the toxicity of nanomaterials and nano-enabled products is important for human and environmental health and safety as well as public acceptance. Assessing the state of knowledge about nanotoxicology is an important step in promoting comprehensive understanding of the health and environmental implications of these new materials. To this end, we employed bibliometric techniques to characterize the prevalence and distribution of the current scientific literature. We found that the nano-toxicological literature is dispersed across a range of disciplines and sub-fields; focused on in vitro testing; often does not specify an exposure pathway; and tends to emphasize acute toxicity and mortality rather than chronic exposure and morbidity.
\end{abstract}

A. D. Ostrowski - T. Martin · J. Conti .

I. Hurt · B. H. Harthorn ( $\square)$

NSF Center for Nanotechnology in Society, University of California, Santa Barbara, USA

e-mail: harthorn@cns.ucsb.edu

\section{A. D. Ostrowski · T. Martin}

Department of Chemistry and Biochemistry, University of California, Santa Barbara, USA

J. Conti

Department of Sociology, University of California,

Santa Barbara, USA

I. Hurt

Department of Geography, University of California,

Santa Barbara, USA
Finally, there is very little research on consumer products, particularly on their environmental fate, and most research is on the toxicity of basic nanomaterials. The implications for toxicologists, regulators and social scientists studying nanotechnology and society are discussed.

Keywords Nanotoxicology - Exposure pathway · Literature review $\cdot$ Environment $\cdot$ Health and safety EHS

\section{Introduction}

Understanding the toxicity of nanomaterials and nano-enabled products is important for human and environmental health and safety as well as public acceptance. The scientific literature is a primary source of information about nanomaterial toxicology and thus plays a role in the emerging dialogue about the safety of nano-enabled products.

There have been several recent reviews, reports, and assessments on the current state and/or challenges of determining the toxicology of nanomaterials (Buzea et al. 2007; Fischer and Chan 2007; Hutchison 2008; Lubick 2008; Maynard et al. 2006; Nel et al. 2006). Most agree that there has been a lack of systematic approaches to assessing the toxicology of nanomaterials and that there is a need for standard methodologies to examine the human health and environmental implications of novel nanomaterial 
characteristics. Hutchison (2008); Fischer and Chan (2007) argue for characterization of the entire nanomaterial life-cycle, paying attention to both exposure pathway and final fate. Lubick (2008) also points to a "deluge of papers" in a broad range of journals that make it difficult to remain informed about the current toxicological results. Extending this line of concern for the state of nanotoxicology, we employed bibliometric techniques to characterize the prevalence and distribution of the current scientific literature, asking the following questions:

- What is the relative distribution of published nanotoxicological research across the areas of human health and the environment?

- Within each of these research domains, what emphases have emerged thus far?

- Which materials and exposure pathways have been researched and which have not?

- Which stages of the nanomaterial life-cycle have been addressed in toxicological research?

In this commentary, we use the above questions to determine base-line measures for the current state of knowledge about nanomaterial toxicity. We highlight apparent gaps in the ongoing nano-toxicological literature that could have significant implications for experts, regulators, and the public. ${ }^{1}$ Assessing the scientific literature is important at this juncture as the toxicology of nanomaterials has increasingly become the subject of regulatory and media attention. ${ }^{2}$

\section{Methods}

To characterize the peer-reviewed literature on nanotoxicology, we utilized two complementary bibliometric strategies. First SciFinder Scholar, a

\footnotetext{
1 We are a research team in a NSF center, the Center for Nanotechnology in Society at University of California at Santa Barbara, which is charged with both understanding and engaging with different interest groups, including the US public. Nanotoxicity questions figure prominently in such engagement; we aimed to see how difficult it is for educated non-specialists to locate and assess the extant literature.

${ }^{2}$ It precedes the roll-out of a NSF and EPA sponsored national Center for Environmental Implications of Nanotechnology, intended to more systematically address and integrate research on the toxicological and ecological effects of nanotechnology. The program solicitation is available at: http://www.nsf.gov/pubs/2007/nsf07590/nsf07590.htm.
}

search engine for scientific literature across the Physical, Biomedical, and Natural Sciences utilizing the CAS (Chemical Abstracts Service) and MEDLINE (U.S. National Library of Medicine) databases were used. ${ }^{3}$ To construct a comprehensive population of journal and review articles in English for the period between 2000 and 2007, we used a modified search term strategy from a list developed by the International Council on Nanotechnology (ICON) at Rice University for their database of research on environmental health and safety (EHS) of nanomaterials. ${ }^{4}$ The search terms included: safety, environmental health, human health, animal health, toxic, nano, nanomaterial, nanoparticle, nanotechnology, Buckyball, fullerene, quantum dot, and ultrafine. We also utilized the ICON EHS database to examine the types of studies that have been researched (e.g., in vivo vs. in vitro) and to compare across nanomaterial types (e.g., carbon, semiconductor, metal, etc.). The selection and categorization of nanomaterial types (e.g., oxides, carbon, etc.) was consistent with that of the ICON database, which categorizes primarily on a chemical basis.

\section{Findings}

Peer-reviewed research on nanomaterials and their toxicology has grown nearly 600 percent since the year 2000, increasing almost exponentially across the 7-year period. As noted by Lubick (2008), the scholarly literature is dispersed across a wide range of disciplines and journals. Our search of SciFinder Scholar produced approximately 900 total articles in about 58 different journals. The journals with the greatest number of relevant articles had 18 articles at most and are spread across Chemistry, Biology, Physics and Engineering fields. This is consistent with the interdisciplinarity of nanotechnology, showing that if one is to stay current on the published

\footnotetext{
${ }^{3}$ The SciFinder Scholar search tool is a product of the CAS division of the American Chemical Society. Information and access to the database is available at: http://www.cas.org/ SCIFINDER/SCHOLAR/index.html.

4 The ICON database is available through Rice University at: http://icon.rice.edu/resources.cfm?doc_id=8597. Last accessed March 2008.
} 


\section{Global Distribution of Nanotoxicology Scientific Literature}

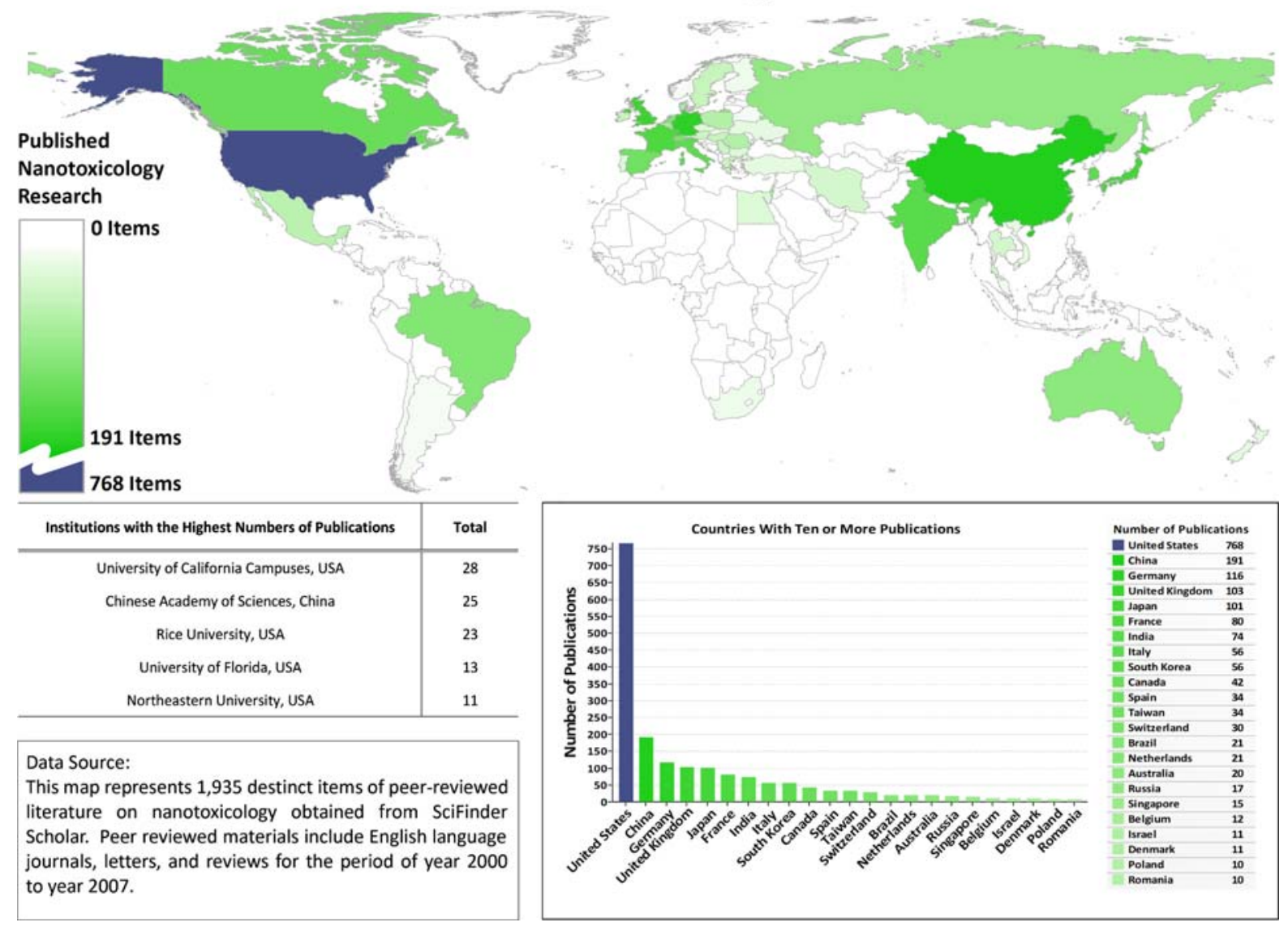

Fig. 1 Global distribution of published nanotoxicology research by institution

literature, it requires maintaining a knowledge base from a variety of different sciences.

The journal articles were also analyzed by country of origin, using GIS plots of the institution at which the research was performed. As shown in Fig. 1, the United States leads in publications on nanotoxicology with almost 550 more publications. China is the second, followed by Germany, the UK, and Japan. This diversity of where the research is performed, along with the interdisciplinary nature of the nanotechnology field, can be one explanation for the variety of approaches and lack of standard methods for studying the toxicity of nanomaterials noted by Lubick (2008); Fischer and Chan (2007).

To characterize the approaches and techniques used to study the toxicology of each nanomaterial, we relied on the ICON EHS database, searching by method of study for each nanomaterial type. While there are differences in the number of studies for each nanomaterial, as shown in Fig. 2, we found that most research has relied on in vitro techniques across all specified material types. This confirms the conclusions of Fischer and Chan (2007), Hutchison (2008), and Lubick (2008), showing that there has been a lack of in vivo studies; even fewer focus on environmental impact and fate. The low number of studies on semiconductors and the absence of any environmental studies on these materials are particularly striking, especially as these materials are closer to commercialization in nano-electronics and currently available in the form of quantum dots for fluorescent tags in cell and mice studies. ${ }^{5}$ This is not surprising, however, considering the far lower costs of in vitro research when compared to the cost of in vivo studies.

5 Available at Evident Technologies, http://www.evidenttech. $\mathrm{com} /$. 
Fig. 2 Number of articles relating to material and type of study

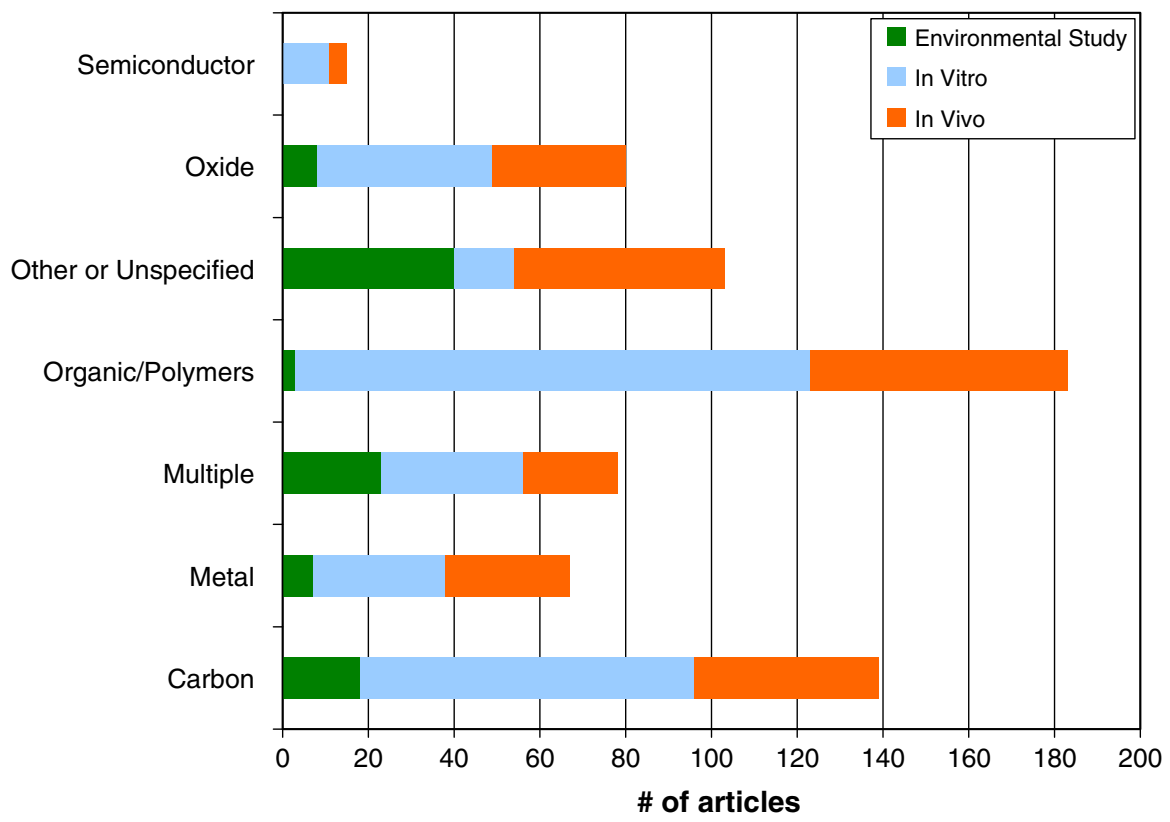

As Fischer and Chan (2007) point out, the toxicology of nanomaterial is likely to change with not only material type, but also the exposure route. The exposure pathway would also determine the dosage, and thus has implications in the toxicity of nanomaterials. To address the question about which exposure pathways are most researched, we again utilized the ICON EHS database to characterize the articles based on material type. Our results show that most research has not specified an exposure pathway (Fig. 3). This may be reflective of the relative infancy of the field, as most studies have been concerned with acute toxicity and not with the complex interactions that would take place in an actual (human) organism. It also points to a predominant concern for traditional dose-dependent mortality without sensitivity to multifaceted and/or chronic exposure hazards and morbidity. When specified, inhalation exposure has been the most researched pathway. This may signal that much work in the field is based on seminal research dealing with exposure to fine and ultrafine particles in the context of air pollution (Oberdörster et al. 2005).

Using ICON EHS database, we also characterized the articles by the cell/organism type that would be affected by the nanomaterials. As shown in Fig. 4, most research has utilized mammalian tissues or organisms. This shows a focus on how the nanomaterial would affect humans or human models (such as mice) and again reflects less overall attention to environmental toxicity. This is particularly noticeable in the low numbers of studies of nanomaterials in soil.

SciFinder Scholar was used to explore what research has been done on nanomaterial life-cycle and to characterize at what stage the toxicity of nanomaterials has been determined. We relied on the categorization of articles by the search engine and assigned categories to one of three stages: Basic Materials, Components or Parts, or Nano-Enabled Products. As shown in Fig. 5, most articles have been concerned with the toxicity of basic materials, rather than intermediate materials or components which incorporate nanoparticles, or nano-enabled consumer products. We found no evidence of toxicological research on the environmental fate of nano-enabled consumer products in the publicly available literature.

\section{Comments and conclusion}

The nano-toxicological literature is dispersed across a range of disciplines and sub-fields. Most of the published research has focused on in vitro testing and does not take into account the complexity of in vivo interactions. Most research has not specified exposure pathway(s) and has focused on acute toxicity and mortality rather than chronic exposure and morbidity. There is very little research on consumer products, particularly on their environmental fate, and most 
Fig. 3 Number of articles by material type and exposure pathway (Collected from ICON)
Fig. 4 Number of articles dealing with ecosystem and material type (Collected from ICON)
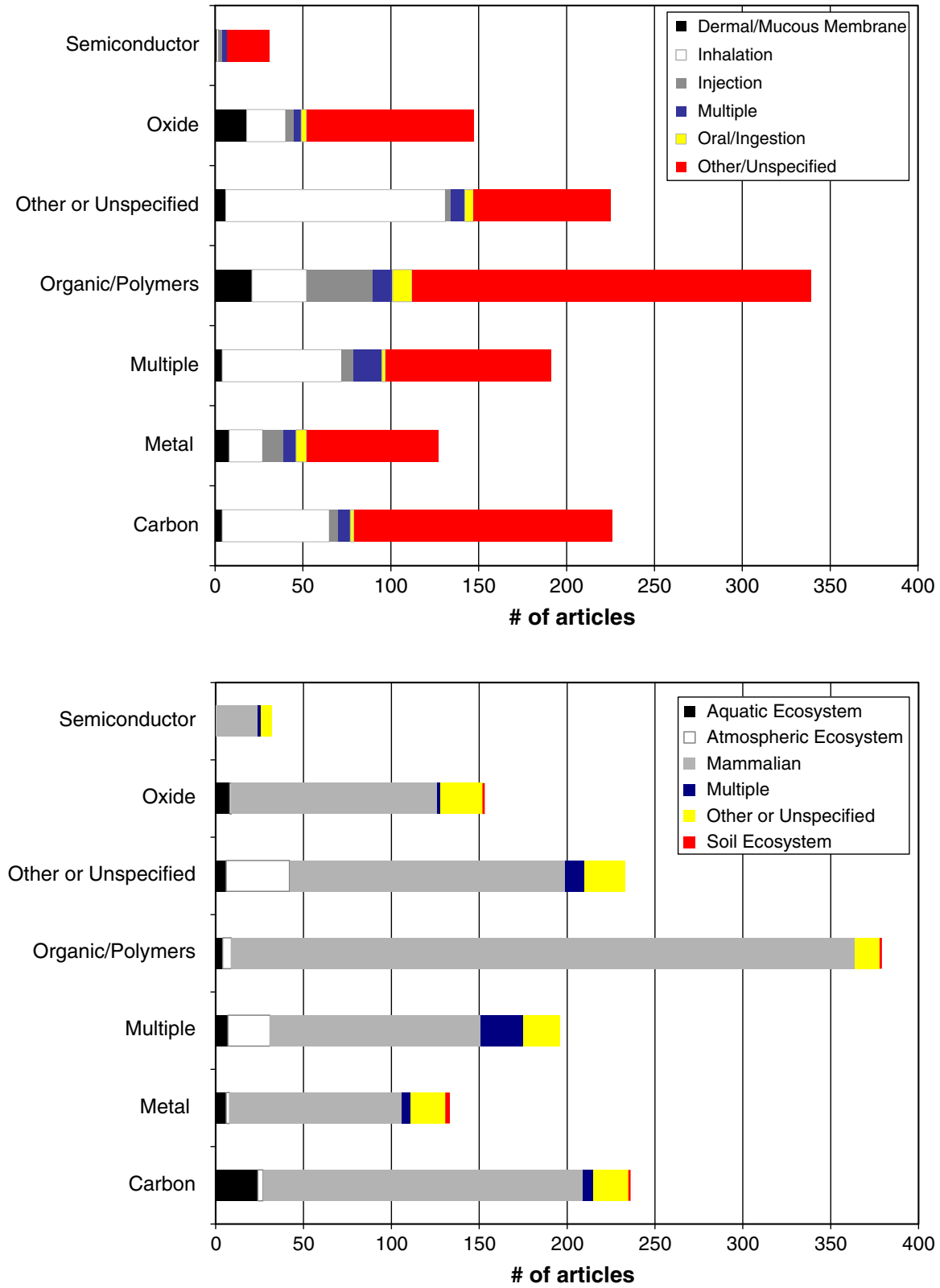

research is on the toxicity of nanomaterials followed by intermediates. This focus on basic materials can be explained by lack of basic understanding, as the nanotechnology field is in its infancy. For example, the differences in behaviors of single-walled carbon nanotubes (SWCNT) and multi-walled carbon nanotubes (MWCNT) remain unclear-especially in a biological environment-and CNTs are among the best studied nanomaterials.

The lack of publicly available research on the toxicity of nano-enabled consumer products and the product end life has implications for public acceptance of nanotechnology. A survey of industrial practices suggests that industrial nanotoxicological testing of consumer products is limited (Conti et al. 2008). It is likely that companies such as DuPont who developed their own nanomaterial testing guidelines ${ }^{6}$ are doing in-house testing. However, the results of

${ }^{6}$ The Nano Risk Framework, developed by DuPont and the Environmental Defense Fund, available at http://www.nanorisk framework.com/. 
Fig. 5 The number of toxicology articles by stage in life-cycle (Collected from SciFinder)

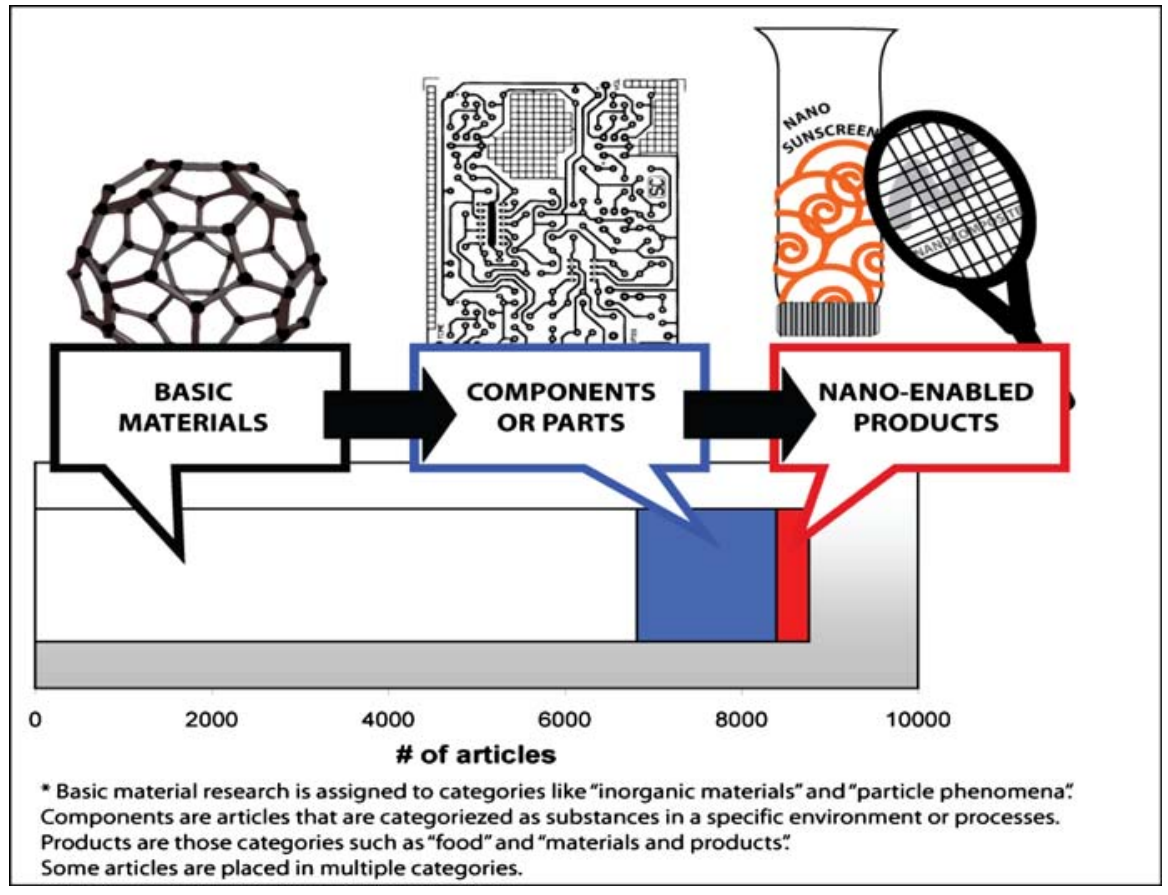

such studies may not be published in the peerreviewed literature characterized here. We would point to a significant risk to the nanotechnology enterprise if hazard data are to come only from industry. The US public is unlikely to trust and accept such assurances, and indeed, such assurances from an un-trusted source are likely to amplify public perception of risk (Poortinga and Pidgeon 2004).

These initial results have significant implications for toxicologists, regulators, and social scientists studying nanotechnology and society. The diffuseness of the scholarly literature may challenge the abilities of the public and civil society to stay informed about the toxicological implications of nanomaterials, as keeping up to date with the literature requires subscription to a proprietary database and not just access to a single or a few journals. It may also make it more difficult to communicate realistic expectations about health and safety to consumers. As described by Hutchison (2008), unknown toxicity of consumer products may pose serious problems for public acceptance of nano-enabled products. With its database, ICON has provided a valuable service in making the references to the scientific literature publicly available; however, a larger, coordinated effort in clearinghouse construction seems to be called for, as access to the journals themselves is necessarily limited to those in academia or industry with collective resources. Maynard et al. (2006) elaborates further by urging for systematic risk research to emerge in all facets, government, industry, academia, and with other stakeholders if nano-industries are to thrive, from nano-materials to nano-enabled products.

This research provides measures for the claims in previous reports on nanomaterial toxicology, demonstrating significant gaps in the emerging field of nanotoxicology. Are these merely the result of the newness or infancy of the field that will be remedied over time as more attention and funding are dedicated to nanotoxicological research? Or, are such gaps the result of specific challenges posed by "nano-scale" materials to established toxicological approaches? Addressing these questions will require additional research, the accessible dissemination of which will contribute to the comprehensive development of the nanotoxicology field.

Acknowledgments This research at the CNS-UCSB is funded by the National Science Foundation under cooperative agreement \#SES 0531184. Any opinions, findings, and conclusions are those of the authors and do not necessarily reflect the views of the National Science Foundation. The authors thank Milind Kandlikar, Trish Holden, and Kristin Kulinowski for helpful discussions during preparation of the manuscript. 


\section{References}

Buzea C, Pacheco II, Robbie K (2007) Nanomaterials and nanoparticles: sources and toxicity. Biointerphases 2:MR17-MR71

Conti JA, Killpack K, Gerritzen G, Huang L, Mircheva M, Delmas M, Harthorn BH, Appelbaum RP, Holden PA (2008) Health and safety practices in the nanomaterials workplace: results from an international survey. Environ Sci Technol 42:3155-3162. doi:10.1021/es702158q

Fischer HC, Chan WCW (2007) Nanotoxicity: the growing need for in vivo study. Curr Opin Biotechnol 18:565-571. doi:10.1016/j.copbio.2007.11.008

Hutchison JE (2008) Greener nanoscience: a proactive approach to advancing applications and reducing implications of nanotechnology. ACS NANO 2:395-402. doi: $10.1021 / \mathrm{nn} 800131 \mathrm{j}$

Lubick N (2008) Risks of nanotechnology remain uncertain. In: Environmental Science \& Technology Online News.
Available at http://pubs.acs.org/subscribe/journals/esthagw/2008/feb/science/nl_nanorisks.html. Accessed $20 \mathrm{Feb}$ 2008

Maynard AD, Aitken RJ, Butz T, Colvin V, Donaldson K, Oberdörster G, Philbert MA, Ryan J, Seaton A, Stone V, Tinkle SS, Tran L, Walker NJ, Warheit DB (2006) Safe handling of nanotechnology. Nature 444:267-269. doi: 10.1038/444267a

Nel A, Xia T, Mädler L, Li N (2006) Toxic potential of materials at the nanolevel. Science 311:622-627. doi: 10.1126/science. 1114397

Oberdörster G, Oberdörster E, Oberdörster J (2005) Nanotoxicology: an emerging discipline evolving from studies of ultrafine particles. Environ Health Perspect 113:823-839

Poortinga W, Pidgeon NF (2004) Trust, the asymmetry principle, and the role of prior beliefs. Risk Anal 24(6):14751486. doi:10.1111/j.0272-4332.2004.00543.x 